\title{
Mechanical Properties Identification of Vulcanized Rubber by Using Mooney-Rivlin Method
}

\author{
Budi Setiyana ${ }^{1, *}$ Rifky Ismail $^{1}$ Jamari Jamari ${ }^{1}$ Sugiyanto ${ }^{1}$ Ismoyo Haryanto ${ }^{1}$ \\ ${ }^{I}$ Mechanical Engineering Department, Faculty of Engineering, University of Diponegoro, Jl. Prof. Soedharto SH, \\ Kampus Tembalang, Semarang 50275, Indonesia \\ *Corresponding author. Email: bsetiyana@yahoo.com
}

\begin{abstract}
Mechanical properties identification of rubber is important to predict rubber performance. Identification of rubber mechanical properties is more difficult to do than it is on metals due to hyper-elastic and nonlinear properties of rubber behavior. Mechanical properties of rubber are often expressed as a Strain Energy Function (SEF). This study investigated mechanical properties identification to obtain the SEF data. The rubber analyzed in this study was vulcanized rubber, both hard and soft compound usually used as tire. Analysis of the tensile test results was conducted based on Mooney-Rivlin method to obtain the SEF data. Verification was carried out by entering the SEF data into numerical simulation. Simulation using FE software was constructed as the friction contact between the rubber and its counter-surface. The results show that the values of stress and friction force in the hard compound is slightly greater than that in the soft compound in the same cases. Fluctuating values was obtained along friction commonly seen in rubber friction. In addition, other mechanical properties can be predicted by obtaining the SEF data such as rubber hardness and rubber deformation when subjected to loading. Therefore, the mechanical performance of the rubber can be easily investigated.
\end{abstract}

Keywords: Mechanical Properties, Strain Energy Function, Vulcanized Rubber.

\section{INTRODUCTION}

Identification of the mechanical properties of rubber is important to predict rubber performance. Due to hyperelastic and nonlinear properties of rubber behavior, identification of rubber mechanical properties is more difficult than that on metals. In metal, the mechanical properties are usually stated as Hooke Law and linear relationship of stress-strain is used. On the other hand, the mechanical properties of rubber are often expressed as a Strain Energy Function (SEF). The Strain (sometimes referred to stored) Energy Function (SEF) describes the amount of elastic energy stored in a unit volume of the materials at a specific state of strain $[1,2]$. However, due to the widespread of rubber properties, Strain Energy Function formulation requires experiment data such as tensile or shear test. It has been noted that in rubber manufacturing, in addition to as a pure rubber, some compounded rubbers are made by incorporating a specified degree of a filled material into rubber material such as carbon black and silica.

There have been some experts to propose the formulation of Strain Energy Function such as Mooney-
Rivlin, Yeoh, Odgen, etc. Some assumptions were made to construct the SEF such as type of rubber material and degree of strain. The SEF model formulation or SEF model contains stored energy, strain and some constants. However, some of the proposed SEF model is difficult to be accomplished particularly to determine some constants value of SEF formulation. The SEF constants or SEF data are determined from SEF model and experimental data analysis [1].

This paper discusses about Mooney-Rivlin model of Strain Energy Function [3,4]. This is one of the wellknown SEF models applied on rubber properties. At first, the SEF model was proposed by Mooney; however, the determination of some constants of SEF is still questionable [3]. To obtain such constants, Rivlin formulation is applied to Mooney SEF and it requires tensile test of rubber specimen [4]. In this research, the rubber analyzed was vulcanized rubber usually used on tire with unknown material composition. Two types of rubber were analyzed in this study, hard compound (vulcanized 1) and soft compound (vulcanized 2). After the constants of the SEF model were obtained, they were 
used as input data in sliding contact simulation between rubber surface and its counter-surface (a rigid indenter). Simulation is carried out by Finite Element Software with some outputs such as stress and friction force [5,6].

\section{METHOD}

This research was started by providing MooneyRivlin model of Strain Energy Function (SEF) and tensile test results of vulcanized rubber specimen. Moreover, Mooney-Rivlin model containing the SEF data or SEF constants is formed by incorporating Rivlin equation regarding the relationship between energy-stress into Mooney SEF model [7]. On the other side, tensile test results were analyzed to find the relationship between true stress and extension ratio. For clearer detail, the road to identify the mechanical properties of rubber material in this research is presented in Figure 1 below.

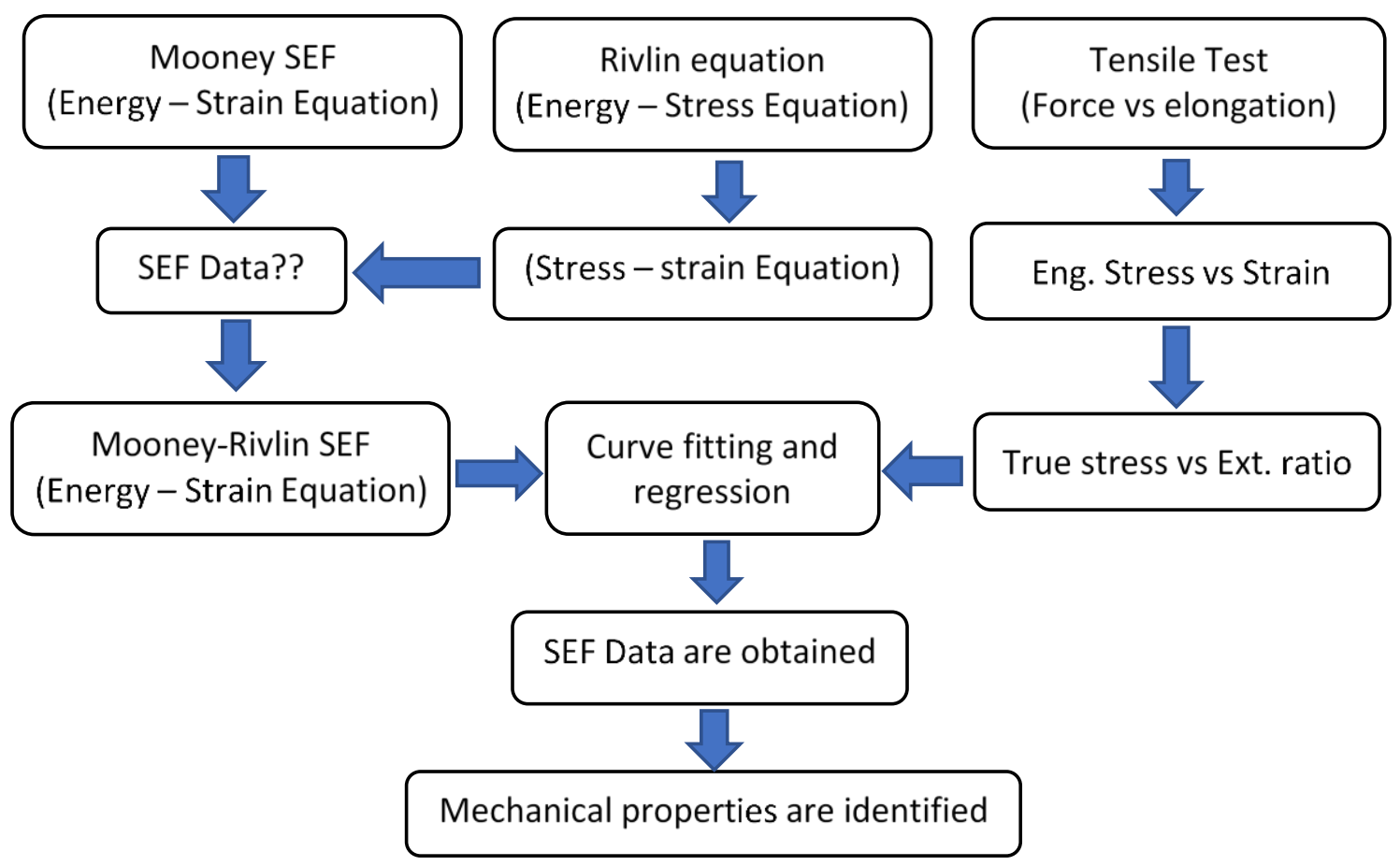

Figure 1 Roadmap to identify the mechanical properties of rubber

Based on the analysed tensile test data and MooneyRivlin equation, a new curve was derived and curve fitting or regression was performed. This process was conducted to obtain SEF constants and to identify mechanical properties. In addition, the resulted SEF constants were used as input data for rubber analysis in Finite Element simulation for verification. Simulation is performed as a static and sliding contact between rubber surface and its counter-surface using a rigid indenter $[8,9]$. Some simulation outputs presented here are such as stress, deformation and friction force.

\section{RESULT AND DISCUSSION}

At first, Mooney proposed Strain Energy Function as presented in Equation (1), in which $\mathrm{W}$ is stored or strain energy, $C_{1}$ and $C_{2}$ are SEF constants, and $I_{1}$ and $I_{2}$ are strain invariant [3]. The SEF constants are obtained analytically and the strain invariant is obtained from the tensile test which depends on the extension ratio $\lambda$ in principal coordinate 1,2 and 3 . The extension ratio is obtained by dividing the final length with initial length of the rubber specimen along tensile test. However, it requires other equation to find out the SEF constants.

$W=C_{1}\left(I_{1}-3\right)+C_{2}\left(I_{2}-3\right)$

$I_{1}=\lambda_{1}^{2}+\lambda_{2}^{2}+\lambda_{3}^{2}$

$I_{2}=\lambda_{1}^{2} \lambda_{2}^{2}+\lambda_{2}^{2} \lambda_{3}^{2}+\lambda_{3}^{2} \lambda_{1}^{2}$

Rivlin's equation which states the relationship between stored energy and stress is applied in this Mooney SEF model. There is an assumption stating that rubber material is incompressible and therefore there is no change on rubber material volume [4]. In this case, reduced stress $\sigma^{*}$ and true stress $\sigma$ are introduced here. Concerning the uniaxial tensile test of the rubber specimen, Mooney-Rivlin equation regarding the SEF constants are given as the following,

$\frac{\sigma^{*}}{2}=\frac{\sigma}{2\left(\lambda-\lambda^{-2}\right)}=C_{1}+\frac{C_{2}}{\lambda}$

From equation (4), it can be observed that this equation needs to perform the plotting of the relationship 
between $\frac{\sigma^{*}}{2}$ versus $\frac{1}{\lambda}$ to find the SEF constants, i. e. $\mathrm{C}_{1}$ and $\mathrm{C}_{2}$. This equation is performed by incorporating the data of stress $\sigma$ and extension ratio $\lambda$ from the tensile test. Thus, the SEF constants can be obtained through curve fitting or linear regression from this relationship. Therefore, it is possible to identify Mooney-Rivlin SEF model for describing the mechanical properties of the rubber.

Figure 2 presents the results of tensile test on vulcanized rubber obtained along with the relationship between engineering stress $\sigma_{e n g}$ associated to the extension ratio $\lambda$. The engineering stress is derived from the tensile force along the testing. Meanwhile, the extension ratio is resulted from the elongation during the testing. Analysis is conducted by considering the incompressibility of rubber material [7]. It can be seen that at large extension ratio, vulcanized 1 provides higher stress than vulcanized 2 .

As viewed in figure 2, the next step to do was to find the relationship between true stress $\sigma$ and extension ratio $\lambda$ by calculating the equation $\sigma=\sigma_{e n g} \lambda$. Therefore, the reduced stress $\sigma^{*}$ can be calculated and the relationship between $\sigma^{*} / 2$ concerning $1 / \lambda$ can be graphically presented based on the true stress and extension ratio $n$ data. The construction was obtained based on equation (4) and this is graphically presented in figure 3.

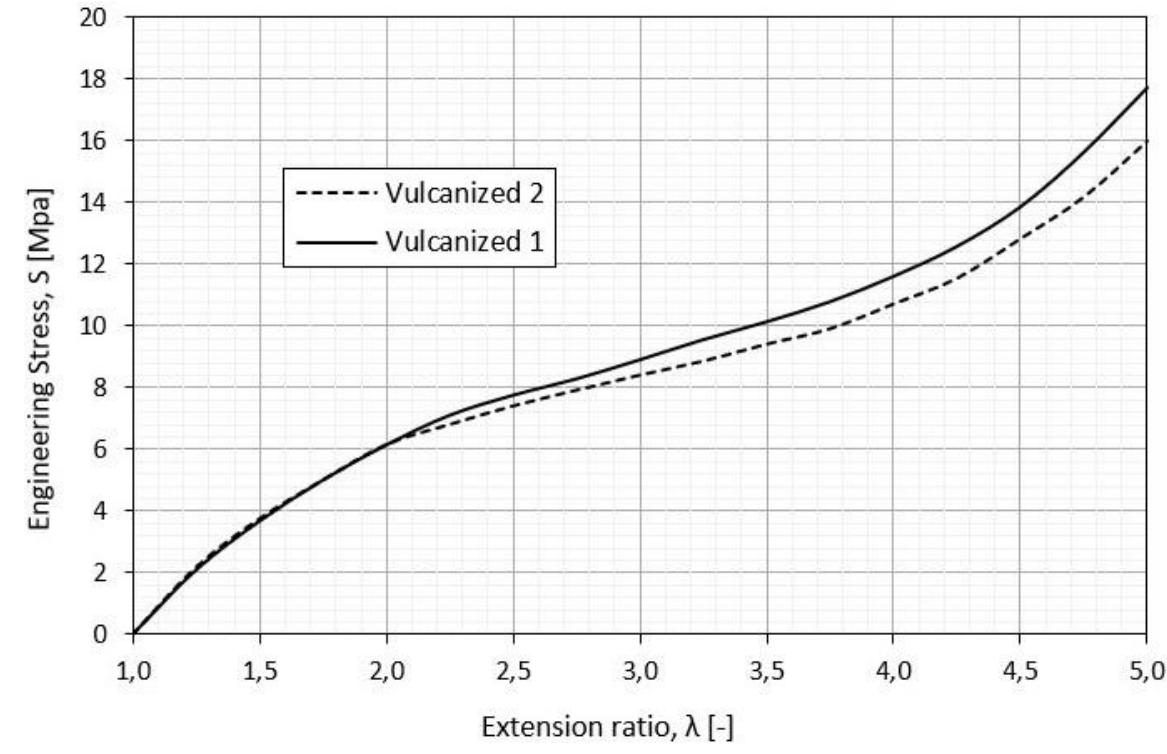

Figure 2 Relationship between engineering stresses regarding extension ratio

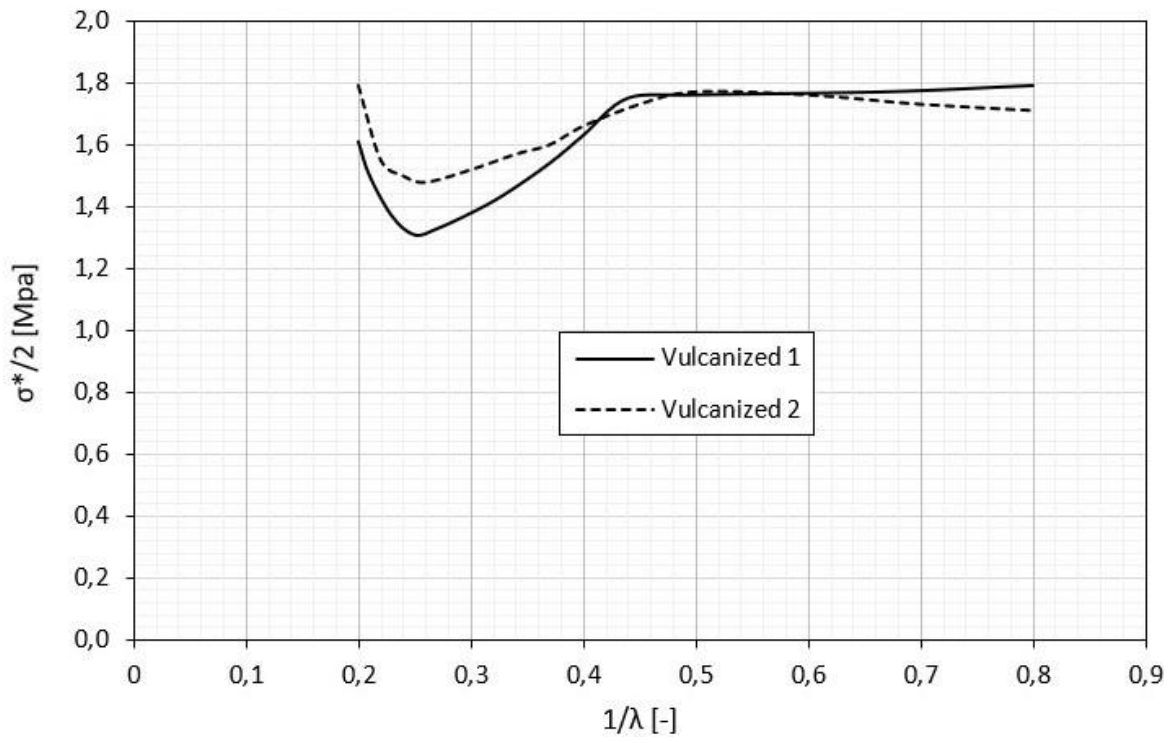

Figure 3 Relationship between $\sigma^{*} / 2$ related to $1 / \lambda$ of vulcanized. 
Based on figure 3 and equation 4 , the constants $C_{1}$ and $\mathrm{C}_{2}$ can be obtained by curve fitting using linear regression. In figure 3 , it can be seen that if horizontal axis is denoted as $\mathrm{X}$ and vertical axis is denoted as $\mathrm{Y}$; thus, elements of $1 / \lambda$ are $X(i)$ and elements of $\sigma^{*} / 2$ are $Y(i)$, which subscript $i$ is the data number. At last, the SEF constants value is calculated using equation 5 and the results are presented in Table 1. It shows that vulcanized 1 has higher intercept $C_{1}$ than that of vulcanized 2 . However, vulcanized 1 has lower gradient $\mathrm{C}_{2}$ than vulcanized 2 .

$$
\begin{aligned}
C_{2} & =\frac{\left(\sum Y i\right)\left(\sum X i^{2}\right)-\left(\sum X i\right)\left(\sum X i Y i\right)}{n \sum X i^{2}-\left(\sum X i\right)^{2}} \\
C_{1} & =\frac{n \sum X i Y i-\left(\sum X i\right)\left(\sum Y i\right)}{n \sum X i^{2}-\left(\sum X i\right)^{2}}
\end{aligned}
$$

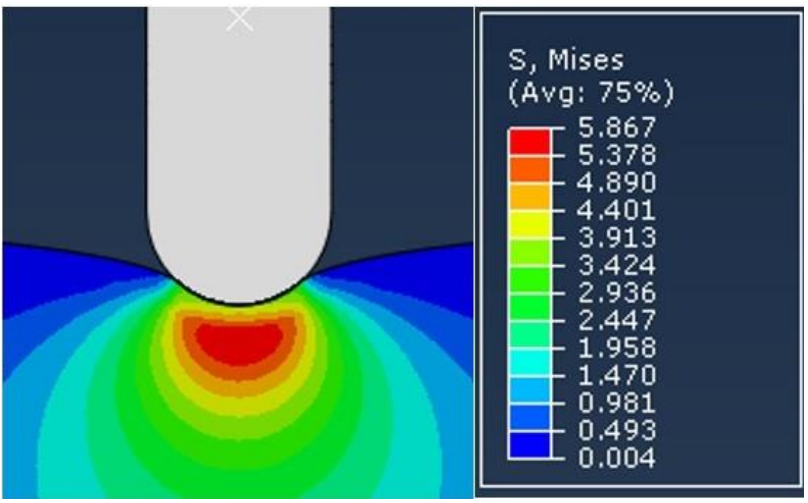

(a)
Table 1. The obtained SEF constants of vulcanized rubber

\begin{tabular}{lcc} 
Materials & $\mathbf{C}_{\mathbf{1}}(\mathbf{M P a})$ & $\mathbf{C}_{\mathbf{2}}(\mathbf{M P a})$ \\
\hline Vulcanized-1 & 0.81 & 1.24 \\
Vulcanized-2 & 0.38 & 1.48 \\
\hline
\end{tabular}

In rubber analysis, the SEF constants were used as input data for rubber behavior simulation. This paper presents Finite Element simulation using commercial software ABAQUS for indentation contact on the rubber surface using a blade rigid indenter. Indentation or penetration as carried out in static as well sliding indentation. The radius of indenter tip is $0.5 \mathrm{~mm}$ and has $5 \mathrm{~mm} / \mathrm{s}$ constant speed along sliding. The simulation outputs were stress pattern and friction phenomena occurring on the rubber surface.

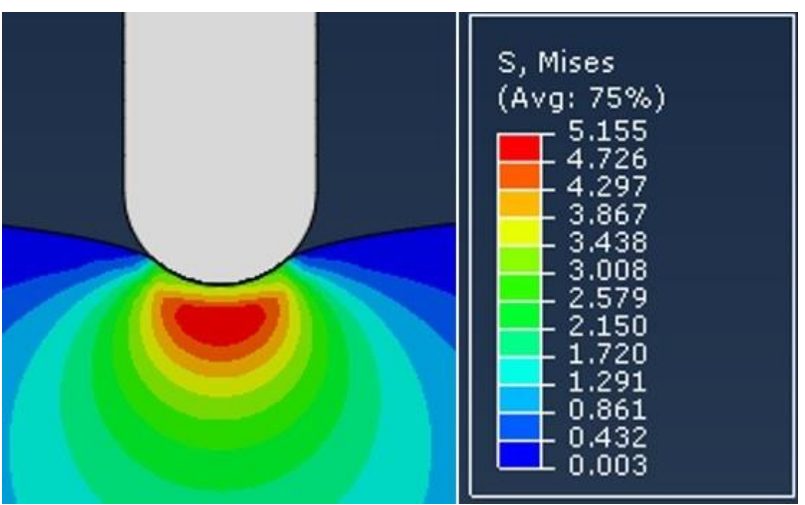

(b)

Figure 4 The von-Mises stress pattern of the vulcanized 1 (a) and vulcanized 2 (b) due to a static indentation with 0.5 mm sliding depth

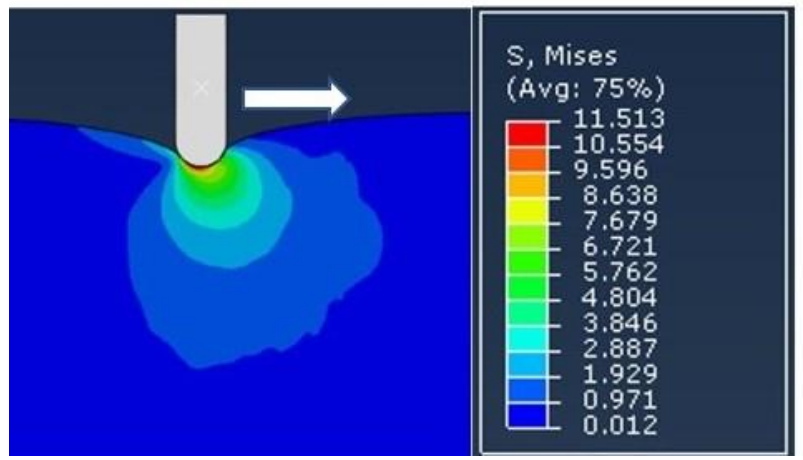

(a)

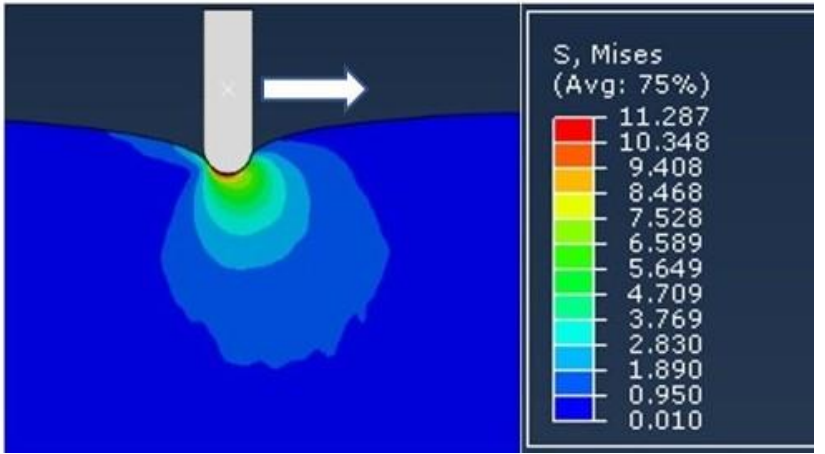

(b)

Figure 5 The von-Mises stress pattern of the vulcanized 1 (a) and vulcanized 2 (b) due to a sliding indentation with $0.5 \mathrm{~mm}$ sliding depth. The arrow indicates the siding direction.

Figure 4 shows the stress pattern on the rubber material due to a static indentation with $0.5 \mathrm{~mm}$ sliding depth. It can be clearly seen that the stress pattern is in symmetrical form between the left and right sides. In addition, it can be logically understood because of symmetrical loading of the indenter which applies on the rubber surface. Figure 5 shows stress pattern on the sliding contact. As seen in figure 5, the maximum stress tends to occur on the front section of sliding direction. Accordingly, it agrees with the rubber abrasion phenomena in which an initial crack emerged on its maximum stress location. 


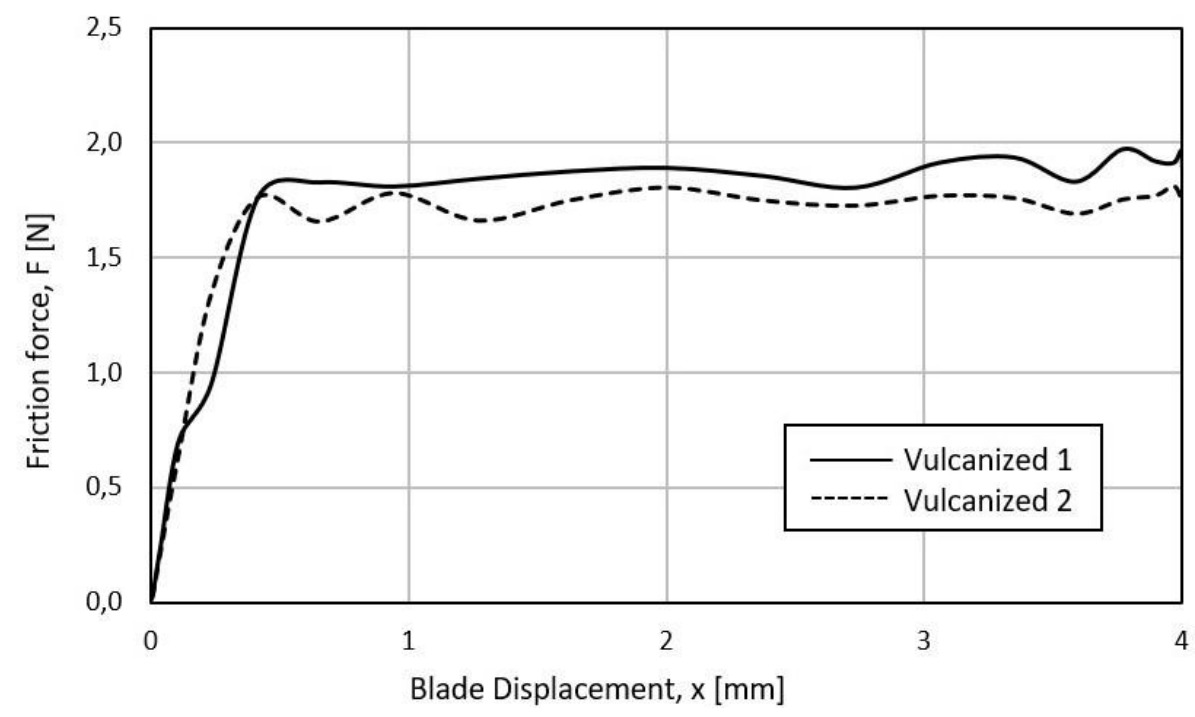

Figure 6 The friction force of the vulcanized rubber along sliding contact using blade indenter with $0.5 \mathrm{~mm}$ sliding depth.

Figure 6 shows friction force along sliding contact obtained by using the blade indenter as the counter surface. Fluctuating friction force was obtained in this study. However, the vulcanized 2 (soft compound) has more fluctuating values than hard compound does. It is possibly due to the low stiffness value of vulcanized 2 (soft compound). In consequence, it is easier to oscillate or vibrate than vulcanized 1 (hard compound).

\section{CONCLUSION}

Mechanical properties identification of vulcanized rubber was carried out by incorporating Rivlin equation into the Strain Energy Function (SEF) of Mooney model. It is then called as Mooney-Rivlin model and the SEF data were investigated by using this model. Based on the results of tensile test on the analyzed rubber specimen and the Mooney-Rivlin model, the SEF data were obtained by using regression method. In addition, the SEF data obtained were applied as input data for rubber behavior simulation using Finite Element software. In the same cases, the vulcanized 1 (hard compound) has a higher stress than vulcanized 2 (soft compound). Moreover, in sliding contact simulation with the rigid counter-surface, vulcanized 2 tends to easier oscillate than vulcanized 1 .

\section{ACKNOWLEDGMENTS}

This study was granted by Engineering Faculty of Diponegoro University, Semarang Indonesia in the scheme of Strategic Research for year of 2020.

\section{REFERENCES}

[1] Gent, A. N. (2013). Rubber Elasticity: Basic Concepts and Behavior. The Science and
Technology of Rubber, 1-26. https://doi.org/10.1016/B978-0-12-3945846.00001-7.

[2] Persson, B. N. J. (1998). On the theory of rubber friction. Surface Science, 401(3), 445-454. https://doi.org/10.1016/S0039-6028(98)00051-X.

[3] Mooney, M. (1940). A theory of large elastic deformation. Journal of Applied Physics, Vol. 11, p. 582 .

[4] Rivlin, R. S. (1948). Large elastic deformations of isotropic materials I. Fundamental concepts. Philosophical Transactions of the Royal Society A, Vol. 240, p. 459-470, 1948.

[5] MSC Software Whitepaper. (2000). Nonlinear finite element analysis of elastomers - technical paper. www.mscsoftware.com.

[6] ABAQUS 6.11. (2010). Abaqus Analysis User's Manual. 15.

[7] Liang, H. (2007). Investigating the Mechanism of Elastomer Abrasion. August, 1-192.

[8] Setiyana, B., Prabowo, C., Jamari, J., Ismail, R., Sugiyanto, S., \& Saputra, E. (2020). Numerical investigation of the hardness of tire rubber material by indentation method. Journal of Physics: Conference Series, 1517(1). https://doi.org/10.1088/1742-6596/1517/1/012020.

[9] Zhang, S. W. (2004). Tribology of Elastomers: Tribology and Interface Engineering Series. Amsterdam: Elsevier. 